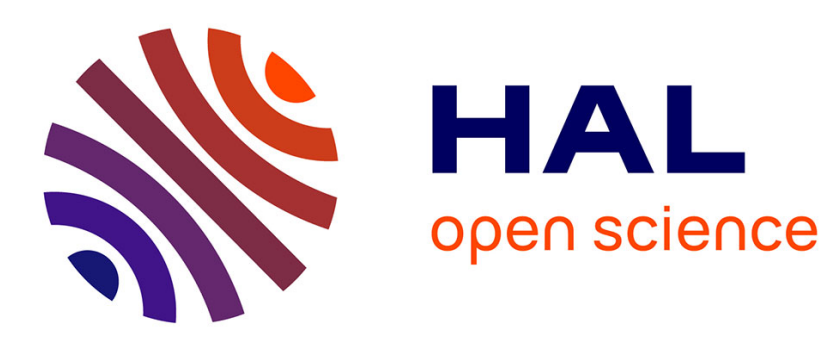

\title{
Foundations of a Simple and Unified Component-Oriented Language
}

Luc Fabresse, Christophe Dony, Marianne Huchard

\section{To cite this version:}

Luc Fabresse, Christophe Dony, Marianne Huchard. Foundations of a Simple and Unified ComponentOriented Language. Computer Languages, Systems and Structures, 2008, 34 (2-3), pp.130-149. 10.1016/j.cl.2007.05.002 . lirmm-00175781

\section{HAL Id: lirmm-00175781 https://hal-lirmm.ccsd.cnrs.fr/lirmm-00175781}

Submitted on 1 Oct 2007

HAL is a multi-disciplinary open access archive for the deposit and dissemination of scientific research documents, whether they are published or not. The documents may come from teaching and research institutions in France or abroad, or from public or private research centers.
L'archive ouverte pluridisciplinaire HAL, est destinée au dépôt et à la diffusion de documents scientifiques de niveau recherche, publiés ou non, émanant des établissements d'enseignement et de recherche français ou étrangers, des laboratoires publics ou privés. 


\section{Author's Accepted Manuscript}

Foundations of a simple and unified componentoriented language

Luc Fabresse, Christophe Dony, Marianne Huchard

PII:

S1477-8424(07)00016-4

DOI:

doi:10.1016/j.cl.2007.05.002

Reference:

COMLAN 67

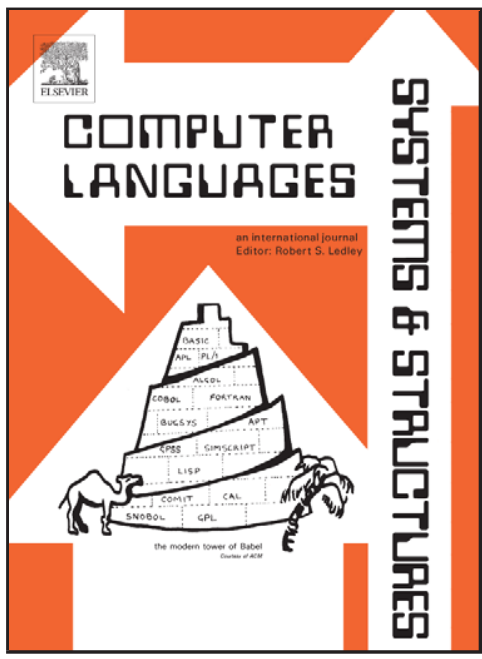

To appear in: $\quad$ Computer Languages Systems \&

www.elsevier.com/locate/cl Structures

Received date: 15 December 2006

Accepted date: 1 May 2007

Cite this article as: Luc Fabresse, Christophe Dony and Marianne Huchard, Foundations of a simple and unified component-oriented language, Computer Languages Systems \& Structures (2007), doi:10.1016/j.cl.2007.05.002

This is a PDF file of an unedited manuscript that has been accepted for publication. As a service to our customers we are providing this early version of the manuscript. The manuscript will undergo copyediting, typesetting, and review of the resulting galley proof before it is published in its final citable form. Please note that during the production process errors may be discovered which could affect the content, and all legal disclaimers that apply to the journal pertain. 


\title{
Foundations of a Simple and Unified Component-Oriented Language
}

\author{
Luc Fabresse, Christophe Dony, Marianne Huchard \\ Lirmm, UMR 5506 CNRS et Université Montpellier II \\ 161, rue Ada \\ 34392 Montpellier Cedex 5 \\ http://www. lirmm.fr \\ \{fabresse,dony,huchard\}@lirmm.fr
}

\begin{abstract}
Component-oriented programming is actually a key research track in software engineering. A variety of component-oriented languages have been proposed with new or adapted abstractions and mechanisms to support this new paradigm. However, the proposed features vary quite widely from one proposal to another. There is a need for a closer analysis and synthesis of these features to really discover the new possibilities of component-oriented programming. In this article we present SCL, our proposition of simple language dedicated to component-oriented programming. Through the presentation of SCL, we discuss and compare the main features of componentoriented languages such as component class, component, interface, port, service or connector. But, these features are not enough to build a component-oriented language. Indeed, unanticipated connection of independently developed components is one of the key issues of component-oriented programming. Most approaches use language primitives or connectors and shared interfaces to connect components. But shared interfaces are in contradiction with the philosophy of independently developed components. The approach of SCL is to provide a uniform component connection mechanism based on special components called connectors. SCL also integrates component properties which enable connections based on component state changes with no requirements of specific code in components.
\end{abstract}

Key words: component-oriented language, component connection, connector, component property 


\section{Introduction}

Component-based software engineering is widely investigated by research and industry. This interest is driven by the promise of improving current software development practices, such as reusability and extensibility, in significant ways $[26,51]$. Although many models, languages and tools have been proposed, it is still difficult to apply component-oriented programming (COP) in practice. Most of proposed languages, such as UML 2.0 [24] or Wright [5,4], are not executable and dedicated to software specification. COP is currently carried out using object-oriented languages which do not offer specific abstractions to ease COP and have to be used in a disciplined way to guarantee a COP style.

Component-based software engineering needs component-oriented languages (COLs) as well as to transform models $[43,15]$ into executables or to write programs by hand. A component-oriented language must offer specific abstractions or mechanisms to write component-based programs [18]. Among the approaches on components, some programming languages have been proposed such as ComponentJ [48], ArchJava [2], Julia/Fractal [9], Lagoona [18], Piccola [1], Picolo [33], Boxscript [32], Keris [55] or Koala [53], in order to support COP. These languages have brought many new or adapted abstractions and mechanisms such as connection, composition, port, interface, connector, service, module, message but their interpretation vary quite widely from one proposal to another. This is quite normal with such an emerging domain, but there is a need for a closer analysis: which mechanisms are essential (basic) and cannot be removed, which ones are (eventually) redundant? Which are the key ones to achieve component composition? To a larger extent, all these questions raise the issue of knowing which constructs and mechanisms are the main identified features of component orientation (by analogy with object orientation).

In this paper, we present SCL that stands for Simple Component Language which is the result of our study and research about component-oriented programming. On the one hand, SCL is built on a minimal set of concepts applied uniformly in order to ease the understanding of the key concepts of component-oriented programming. The main features of SCL come from existing languages but we argue that we chose the fundamental ones. On the other hand, SCL integrates a new powerful, extensible and uniform component connection mechanism that addresses one of the key issues of component-oriented programming which is the unanticipated connection of independently developed components. In existing languages, the connection mechanism is either a fixed language primitive such as in Fractal [9] or relies on first-class entities named connectors $[49,36]$ which represent connections such as in ArchJava [2] or Sofa [7]. In SCL, a connector is a kind of component dedicated to the adap- 
tation of the communications between components. The SCL connectors offer better decoupling between the reusable business code inside components and the gluing connection code inside connectors. The SCL connection mechanism is based on connectors and enables independently developed components to communicate following different protocols without requiring any special code in components. For example, we propose connectors that offers some possibilities available in aspect-oriented programming [30]. SCL also provide connectors to establish communications based on the publish-subscribe protocol between components without requiring any special code in the publisher or the subscriber component. This is possible because SCL components are defined using the concept of property to externalize component state without breaking component encapsulation. Properties are the support of a new kind of component communication based on changes of property state. We choose Squeak, a Smalltalk implementation, to implement SCL because it is a dynamic language that offers a suitable meta-object protocol that can be easily extended and because we want to provide an easily extensible language.

The paper is organized as follows. Section 2 presents the general context of component-oriented programming. Section 3 discusses if a class-based or a prototype-based approach is suitable for a COL. Section 4 motivates the choice of the core features of SCL. Section 5 explains the service invocation mechanism. Section 6 describes how components can be connected. Section 7 shows that separation of concerns is possible in SCL. Section 8 explains why publish/subscribe communications are an issue in COP and proposes a solution based on properties. Section 9 describes the current implementation of the SCL prototype in Squeak. Section 10 compares SCL features to those existing ones in various COLs and presents some related work. Finally, section 11 concludes and presents future work.

\section{Component-Oriented Programming: What, Why and How?}

COP is based on the idea stating that a software can be built by plugging pieces of software called components. The term "component" has different meanings to many different people depending on the perspective taken. For example, design patterns [19], functions or procedures [34], modules [18], application frameworks [51], object classes [25], and whole applications [37] can be considered as components. Similarly, there are many different definitions for the term component given in the literature [8,23,51]. The component definition reached by consensus is: "A software component is a unit of composition with contractually specified interfaces and explicit context dependencies only. A software component can be deployed independently and is subject to composition by third parties" [51]. 
Originally, researches on component-based software development (CBSD) have been driven by the will of reducing software development costs by increasing software reuse. Indeed, instead of developing new components from scratch, CBSD recommends the reuse of existing components that have already been developed and tested. This idea is expected to reduce development costs because it reduces the development time. A second motivation for componentbased software development has emerged, the will of reducing the evolution cost of a software. This motivation is now more important than the originally one, because software are not developed one time, but continuously developed in order to correct bugs (maintenance) or to add new features (extension). Software evolution is a challenge because of new factors such as the growing software size or the software distribution. Component-based applications, built out of interconnected components, are expected to be easier to evolve. This is because the software evolution of component-based software relies on decoupled components that can evolve independently. This property is named the independent extensibility [51] of component-based software.

New software development methods dedicated to CBSD, such as Kobra [6] or Catalysis [14], focus on the reuse in the earlier steps of the development process. Reuse at the design phase can be considered from two different complementary perspectives: design for reuse or design by reuse. Design for reuse deals with identifying, specifying reusable elements, and integrating them in a reuse system. Design by reuse aims to define new systems engineering processes, and develop tools supporting systematic reuse of components to build new systems. Figure 1 shows that these two complementary concerns are generally targeted by two different actors of the development process: the programmer and the architect. A component-oriented language must offer mechanisms for both of them, without forgetting their respective role: the programmer builds reusable components (for reuse) and the architect builds applications by reusing components (by reuse).

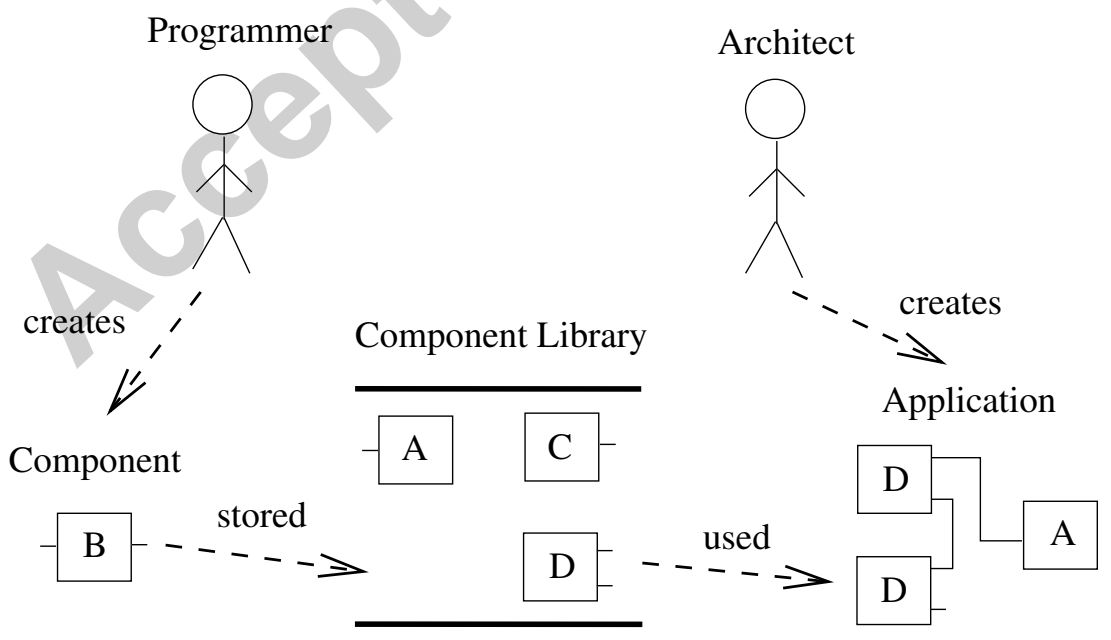

Fig. 1. A high-level view of component-based software development. 


\section{Component-Oriented Languages: Prototype-based or Class- based Languages ?}

In object-oriented languages, the terms "class" and "instance" allow programmers to refer without ambiguity respectively to object descriptions in code and to objects themselves as runtime entities. Although many component-based languages are generally built on a class/instance conceptual model, few of them specify the terms to denote respectively component descriptors (classes) and component themselves (objects). For example, the two keywords component class in ArchJava and component in ComponentJ both denote a component class which can be instantiated. In ArchJava, a component class is an extension of the Java class concept used to define a type of component. A few COLs have been proposed with a prototype-based model (i.e without descriptors). For example, a prototype-based language has been proposed [54] on the top of Java in order to provide primitives to dynamically build, extend and compose software components from Java objects. We think that the arguments for (or against) the use of classes are similar in the component and object worlds and that both approaches are worth to be considered [13].

In SCL, we have chosen a class/instance approach but we clearly distinguish these two concepts. A component is a runtime entity and it is an instance of a component descriptor. Component descriptors are written by the component programmer in order to create off-the-shelf reusable pieces of software while the software architect creates an application by choosing and instantiating some component descriptors and then connecting components (i.e. instances).

\section{Basic Component Structure}

It is largely admitted that a "component can only be accessed through well defined interfaces" [51]. Component interfaces shield the component from its environment and increase its context independence needed by the design for reuse. Component-based languages propose different concepts to describe component interfaces such as services, ports, interfaces, protocols, etc. In different component languages, these words may have different meanings. For example, in Fractal [9] or Enterprise JavaBeans [39], the port and interface concept are mixed that is why they only speak about interfaces. In UML components [12], both port and interface concepts exist such as in ArchJava [2] where interfaces are called port interfaces. That is why we choose to clearly explain the choices that we made for SCL. In SCL, a component provides or requires services through ports described by interfaces. 


\subsection{Services}

A component provides functionalities which are named services. Basically, a service is a subprogram defined in a component, such as a method in the object-oriented model. The main difference between a component and an object is that a component also expresses the services that it requires from other components in order to provide its own ones. Thus, a component has two sets of services: its provided services and its required services. For example, a password manager component may provide services such as generatePwd:, generateALowerCasePwd:, isValid: and require a getRandomNumber service.

\subsection{Ports}

"A component is a static abstraction with plugs" [41]. Ports represent these plugs and are the interaction points [51] of components. This means that all that is possible on a component, such as service invocation, have to be done through its ports. The port construct is present in almost all component models but with different semantics. In component models that support ports, they are either unidirectional such as in or bi-directional. Through unidirectional ports such as in ComponentJ [48] or Fractal [9], a component provides or requires a set of services through its ports. In ArchJava [2] or UML 2.0 [12], ports are bi-directional and a component both requires and provides services through each of its ports.

Unidirectional ports allow the programmer to group some services in a set and require or provide this set. Two kind of unidirectional ports are distinguished: required ports through which the component requires a set of services, and provided ports through which the component provides a set of services. Ports help the programmer to group related services and then defines view points or security policies. Required ports define view points for the component on its environment while provided ports define view points on the component for its environment. A port also defines a security policy because a component that communicates with another component through one of its ports can only access the services accessible through this port. Grouping some required services on required port is also used by programmer to express that these services should be provided by the same component. For example, a component that requires the services: pop and push: through one of its required ports expects that these two services will be executed by the same component.

Bi-directional ports offer the same capabilities than unidirectional ones but they allow the programmer to define more complex point of views than unidirectional ports. Indeed, a bi-directional port describe the role that the com- 
ponent could accomplish in a collaboration. For example, a component may requires a getPwd service and provides open and close services through the same port. This port express that the two services open and close will be provided to a component that itself provides the getPwd service. It is possible to express more accurately the dependencies between services using bi-directional ports.

We choose to integrate unidirectional ports in SCL. On the one hand, this is because they are simpler to understand and to use. The programmer defines services in the component implementation and choose to provide some of them through the provided ports of the component. In SCL, a service may be provided by more than port. On the other hand, this is because it is possible to construct bidirectional ports using unidirectional ones as we will see with the property concept of SCL in section 8. In SCL, a port has a name (a component can not have two ports with the same name). Figure 2 shows the SCL code needed to create a component descriptor, declare ports and instantiate a component.

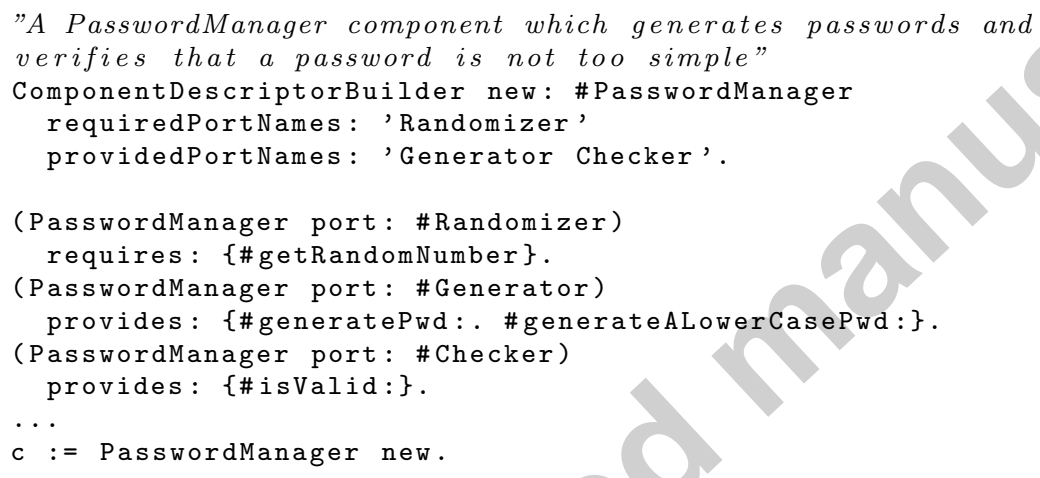

Fig. 2. Definition and instantiation of a component descriptor in SCL.

It is important to note that SCL uses the Smalltalk syntax but the constructs have not always the same meaning than in Smalltalk (it will be shown in section 5). In the above example, a PASSWORdMANAGER is defined with three ports: the required Randomizer port through which the component requires a getRandomNumber service, the Generator and Checker ports that respectively provide services for generating passwords and services for checking passwords. The implementations of the component services are not shown in this example. ComponentDescriptorBuilder is the bootstrap component of ScL. This component is used to create empty component descriptors by using, for example, its new:requiredPortNames:providedPortNames: service. A component descriptor can be completed in order to describe its instances. Finally, the instantiation of a component descriptor is achieved using its new service. 


\subsection{Interfaces}

The "interface" word as many different meanings in the object world and the component world. For example, Fractal [9] distinguishes component interfaces and language interfaces (i.e Java interfaces) but they only keep the term of interface which could be misunderstood. The interface concept is almost in all component models but sometimes mixed with the port concept such as in Fractal [9] or sometimes completely independent such as in UML [12]. Interfaces vary from informal texts descriptions in natural languages to formal descriptions such as in WRIGHT [5]. We distinguish two kind of descriptions: syntactic and semantic descriptions. Syntactical descriptions are generally represented by a named type describing a set of method signatures such Java interfaces [22]. Semantical descriptions are harder to define and are often based on formal theory, such as CSP in WRIGHT [5] or protocols in Sofa [46]. Protocols allow component programmers to define the valid sequences of service invocations through regular expressions. For example, if a port provides three services related to network communications, protocols can be used to describe that the open: service must be invoked first and one time, then the send: service and finally the close service must be invoked to finish the interaction.

Interface compatibility. In ArchJava [3], ComponentJ [48], Fractal [9], a port that requires an interface $I_{1}$ can only be used by a port that provides an interface $I_{2}$ where the type defined by $I_{1}$ is a super-type of the one defined by $I_{2}$. Validation is achieved through typing rules on interfaces: "[...] types stand for semantical specification. While the conformance of an implementation to a behavioral specification cannot be easily checked by current compilers, type conformance is checkable. By simply comparing names, compilers can check that several parties refer to the same standard specification." [10]. Using these kind of interfaces implies that independently developed software components have to be defined using type-compatible interfaces in order to communicate. Depending on the type-compatible relation between interfaces, it will break the independently developed property of components. It is exactly what happen in most component-oriented languages that are Java extensions because they rely on the Java type system which is a named type system in which the subtyping relation of interfaces is explicitly declared in their definition. Structural type systems [11] offer better decoupling since the subtype relationship is computed from the structure of interfaces. But structural type systems are less expressive than named type systems (such as Java). For example, expressing that "a component requires an Stack" is more expressive than expressing that "a component requires two services push: and pop" because it not sure in the second case that is exactly a stack that is needed. In the first case, there is a need for a global stack definition and the defined component will only able to communicate with components that have been defined using the same stack definition such as "a component that provides the stack capabilities". 
In SCL, an interface describes the possible interactions that can be achieved through a given port. Interfaces document the component and enable the automatic validation (static or dynamic) of the component uses. SCL interfaces can be as complex as we could imagine in order to capture the semantical aspects of components. The most basic and necessary interfaces are service signature sets and a programmer defines the interfaces of provided ports in order to choose the services that he wants to provide through a port. But, the interfaces of required ports can be automatically set up on a component during its instantiation by analyzing the implementation of its provided services. Nevertheless, it is hidden for the architect that only knows the external features of component i.e its ports and their associated interface as shown by Figure 3.

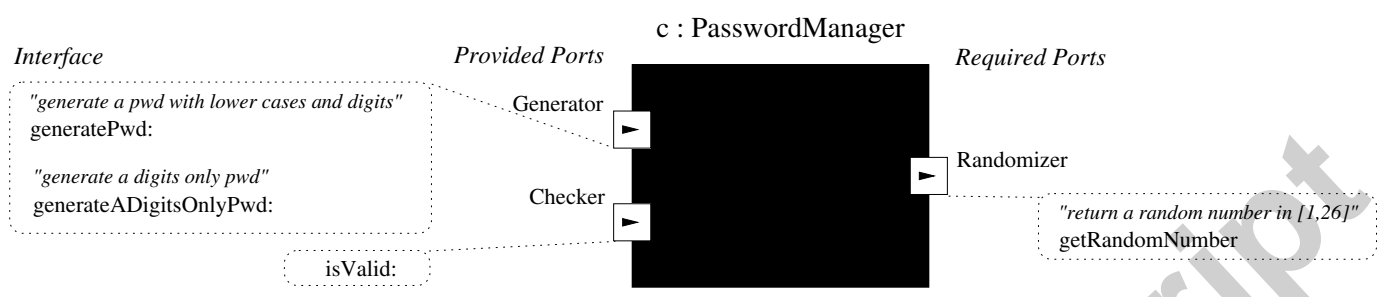

Fig. 3. The architect vision of a component $\mathrm{c}$, instance of the component descriptor Passwordmanager. Ports represented by squares on the component boundary. Triangles designate the direction of service invocations.

\section{Service invocation}

In SCL, components communicate by service invocation through their ports. The syntax and semantics of service invocation it not clearly defined in existing COLs as the message sending mechanism is, in the object world. It is certainly because COLs are object-oriented language extensions such as ArchJava or ComponentJ and uses the message sending mechanism.

A service invocation is similar to a message, it has a receiver, a selector and arguments. But the receiver of a service invocation is a port that indicates through which port the service invocation is done and the selector a service name. Figure 4 shows examples of service invocations. Since SCL uses the Smalltalk syntax, the space character is used for service invocation which the same as message sending but the mechanism is not the same as it has been explained. If the receiver port is a required port of a component c (e.g on line 20 of Figure 4), the invocation will be treated by another component that will be known at connection time (cf. section 6). The invocation of a required service of a component supposes that this component has been connected to other components that provide this service. If the receiver is a provided port of a component $\mathrm{c}$, a service with a matching selector and defined on the 
component description of $\mathrm{c}$, is executed. For example, the service invocation on the line 7 of Figure 4 will produce the execution of the provided service isValid: If there is no corresponding service, the doesNotUnderstand: service of $c$ is executed with the invocation as argument. As all Smalltalk objects respond to the doesNotUnderstand: message, all SCL components own the doesNotUnderstand: service.

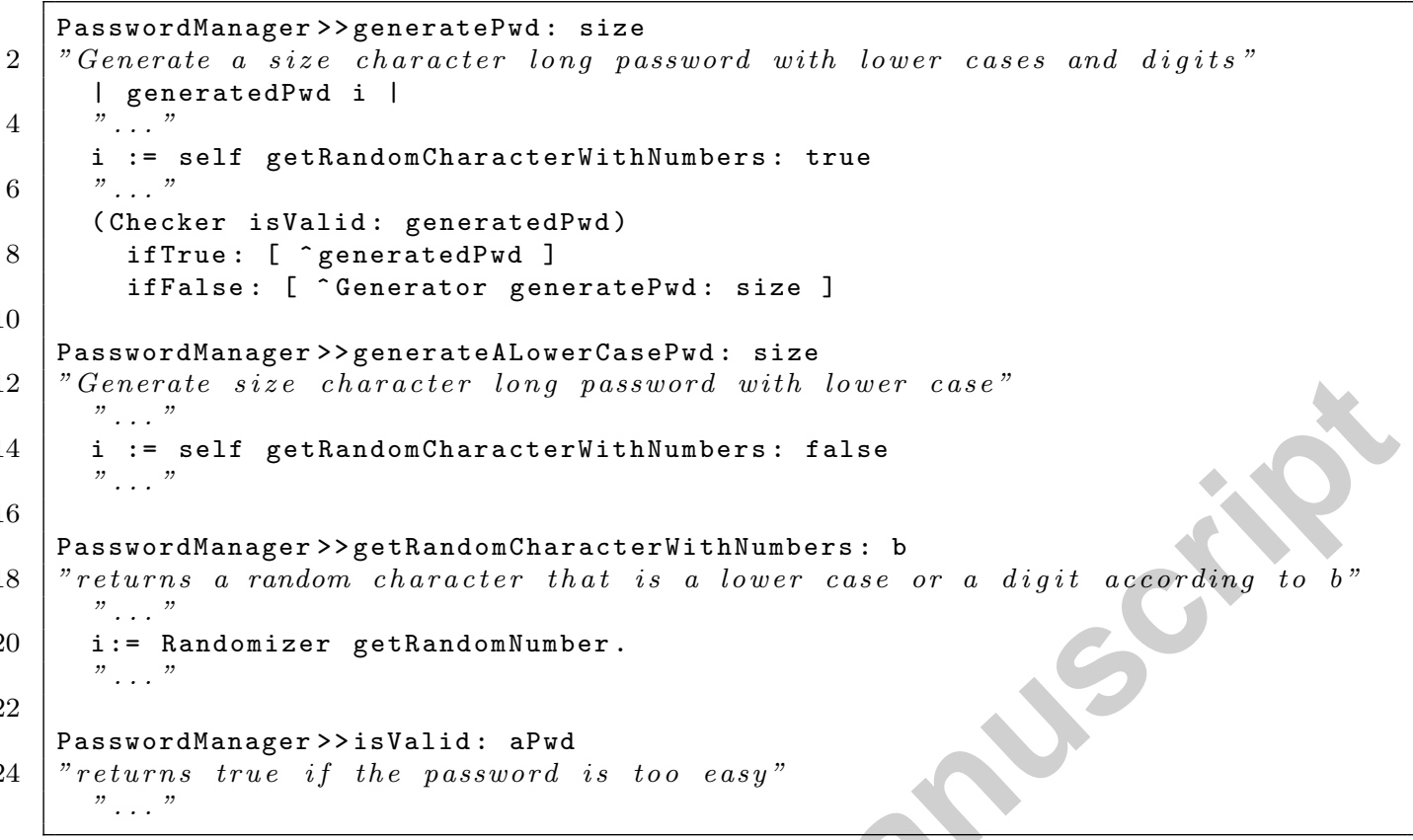

Fig. 4. Partial implementations of the PASSWORdMANAGER services.

Internal services invocations. How to invoke a service that is defined but not provided by any port of the component such as the getRandomCharacterWithNumbers: service? We call internal services, these kind of services. They are useful to organize the internal code of a component but do not be provided. Since these services are not provided nor required, it is not possible to invoke them through a port. We are not aware that some COLs have raised the question: What is self in a COL? In SCL, all components have an internal provided port named self. An internal port or private port can not be accessed outside of its component. The self port of a component automatically provides all the services that are defined in its componentDescriptor. The invocations on lines 5 and 14 of Figure 4 are then a regular service invocations.

Default port. We add that all components have port named default that provides all the provided services of the component. The services provided by this port can be restricted. This port is convenient because when a component is used as the receiver of a service invocation, this is exactly the same as if its default provided port have been used (e.g Figure 5). 
c : = PasswordManager new.

c isValid: 'aaaa'.

$\mathrm{p}:=\mathrm{c}$ generatePwd: 4 .

Fig. 5. Service invocations through the default port of a component.

\section{Component Connection}

COLs propose one main mechanism for component-oriented programming: the connection of components. A component $c$ can invoke the services of another component $d$, only if $c$ and $d$ are connected. Components communications are possible by component connections. Unanticipated is the key-adjective attached to connection that makes component-based software worthwhile. Unanticipated means that a programmer defines a component $c$ with a design for reuse goal, and he must make no other assumptions than what he declares in the interfaces of $c$. The programmer does not know the concrete components that will be connected to $c$, later on by the architect. Another mechanism is proposed by COLs: the composition of components. Composition is used to create a new component called a composite out of existing components. In this section, we present these two mechanisms of connection and composition. We also explain that composition is just a subtle variation of the connection mechanism.

\subsection{Connection}

The connection mechanism connects components through their ports. It is often said that two components $c_{1}$ and $c_{2}$ are connected if at least on port of $c_{1}$ is connected to one port of $c_{2}$. Connecting components require to able to connect ports. This could be achieved using language primitives or using firstclass entities named connectors. Connectors are architectural building blocks used to model interactions among components and rules that govern those interactions [49]. The connection mechanism is provided through various forms and semantics in actual COLs. Let us examine the two solutions of ArchJava and Fractal that we inspired our SCL solution:

ArchJava [3] provides a connect primitive that takes a set of bi-directional ports of components.

connect c_1.p_1, c_2. __ $_{-} 2, \ldots, c_{-} n \cdot p_{-} n$;

Semantics of this connect primitive is that when a required service of a component $c_{i}$ is invoked through its port $p_{i}$, the service to be executed is searched in the set of services provided by ports $p_{1}, \ldots, p_{n}$. If the connect instruction has not failed, there is exactly one compatible service. The ArchJava mechanism is useful to easily express n-ary connections. ArchJava also 
allow the architect to defines its own connector classes that can be used to fix the connection semantics.

$$
\text { connect c_1.p_1, c_2.p_2 with TCPConnector; }
$$

Fractal [9] provides a binding primitive named bindFc that binds one required port (named a client interface in Fractal) with one provided port (named a server interface in Fractal).

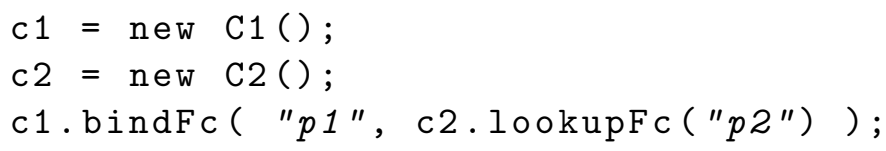

More complex connections can be achieved in Fractal with using binding components. "A binding component is a normal Fractal component whose role is dedicated to communication between components. Binding components are also called connectors: hence Fractal does support connectors, although this concept is not a core concept here, as component or interface." [9]. In Fractal, n-ary connections requires that the architect defines a binding component with exactly the right number of ports. It seems difficult with this connection mechanism to support dynamic n-ary connections.

Before presenting the SCL connection mechanism, it is important to note that a connection mechanism must address mismatches. Connection mismatches are an identified consequence of unanticipated connections [47]. These mismatches occur when we want to connect components that semantically fit well but their connection is not possible because they are not plug-compatible. Mismatches can be solved in whole generality by defining dedicated components as specified by the Adapter design pattern [19]. Another solution is to put glue code in connections (e.g in connectors) in order to adapt components communications. A connection mechanism must tackle this issue and makes the definition of adapters easy or useless thanks to glue code.

In SCL, the connection mechanism relies on connectors. Since we want SCL to be a simple language, we choose that a connector is a component whose role is dedicated to communication between components such as binding components in Fractal. SCL connectors help to solve mismatches problems easily and enable the definition of n-ary connections. All connectors have the same form as Connector that is shown on Figure 6. A connector is composed of two sets of ports named sources and targets, and glue code that uses these ports to establish the connection. All the service invocations sent through source port will be treated by the glue code of the connector.

Figure 7 shows an example of binary connection between a PASSWORDMANAGER component and a RANDOMNumBERGENERATOR component. This connection satisfies the required service of the PASSWORDMANAGER through its Randomizer port, using the service rand provided by the RANDOMNUMBERGENERATOR through its Generator port. Since connectors are regular com- 


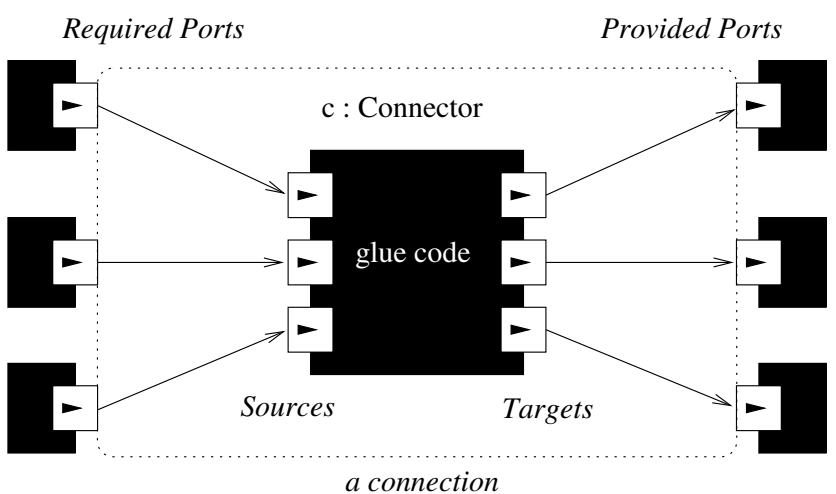

Fig. 6. The general form of a SCL connector

ponents, the architect has to define a component descriptor for the connector that is used to establish the connection. However, SCL proposes simpler syntaxes. For example by instantiating an existing ScL connector descriptor, such as BINARYCONNECTOR, and adapting it by setting its sources, its targets and the glue code, as shown in Figure 8.

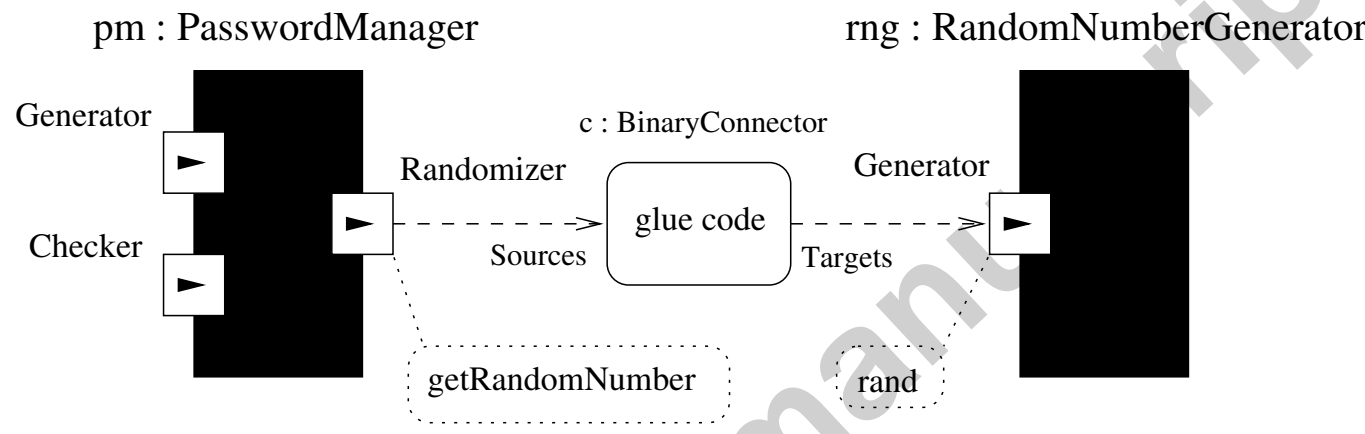

Fig. 7. A binary connection between two components in SCL

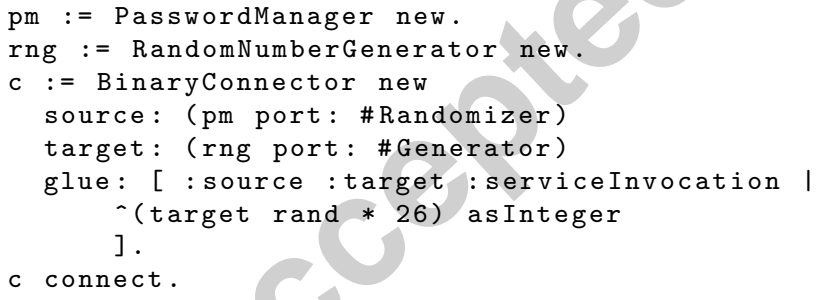

Fig. 8. Connection of two components using a customized BinaryConneCtor

Glue code is represented here by a Smalltalk block [21]. The parameters of this block are always the sources, the targets and the current service invocation that has been received by a source port and must be transmitted through a target port. Of course, a BinARYCONNECTOR has only one source and one target. In the glue code of this example, the result of the rand service is adapted since the getRandomNumber is expected to return a number in the interval $[0,26]$ while the rand service returns a number in the interval 
$[0,1]$. Despite of the fact that this is a simple example, it is important to note that connecting independently developed software components must deal with these kinds of problems. SCL connectors tackle these adaptation issues thanks to glue code that can not be written in components. The last line of code activates the connection by invoking the connect service of the connector $c$ which makes him to execute glue code for each invocation received through one of its source ports. Figure 9 shows some convenient syntax for simple connections. In the two first connections, a BINARYConNECTOR is instantiated with the default behavior that forwards all service invocations coming from the source port to the target port and to return the result back. In the third connection, a TCPCONNECTOR is used to connect these two ports.

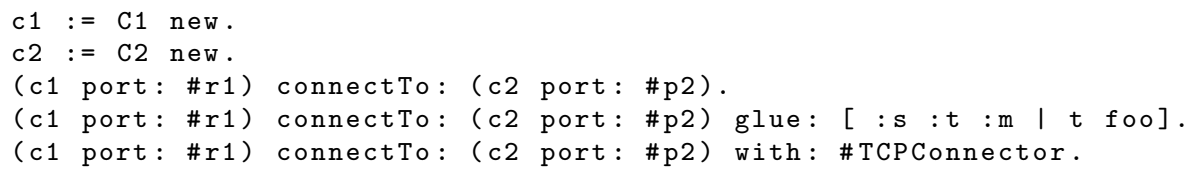

Fig. 9. User friendly definitions of simple connections in SCL

We propose in SCL a small library of connectors based on the taxonomy of software connectors [36] that has eight kinds of connectors have been identified. For example, the BinARYCONNECTOR is a connector of the call connector family that is dedicated to service invocation connections. In this family, there is for example, the BroAdCASTERCONNECTOR that broadcasts each service invocation to all targets, or the FirstResultConnector that returns the first non-nil result by sending invocation successively to each target. Of course, new connectors can be defined.

\subsection{Composition}

The composition mechanism is used in COLs to build composite components out of components and connections. Composite components are useful to abstract over complex systems, provide bigger reusable software entities that hide implementation details. All recent component models $[9,2,48]$ provide a composition mechanism generaly based on the connection mechanism to create composite. This mechanism is provided through various forms in existing languages, e.g the compose primitive in ComponentJ [48], composite components in Fractal or aggregation and containment in (D)COM [37].

Composition is related to encapsulation in the component world. For example, Figure 10 shows an example of three connected components $c_{1}, c_{2}$ and $c_{3}$.

If these components were objects, we could say that $c_{3}$ is a composite object that contains $c_{2}$. In the component world, this is not true because $c_{2}$ may be accessible to other components than $c_{3}$. The components used in a composite 


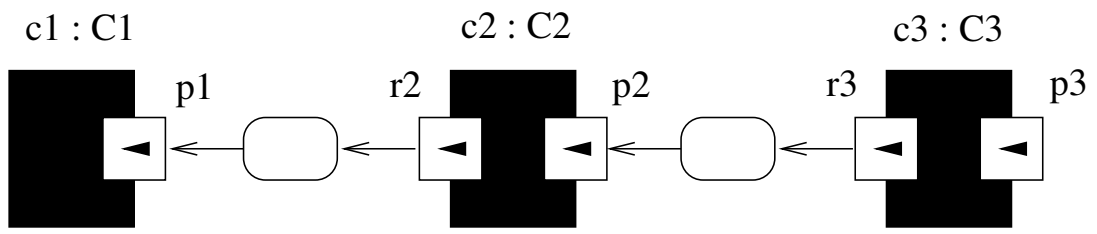

Fig. 10. Three connected components in SCL

(also called its sub-components) are internal and exclusive to their composite. In our example, $c_{3}$ can be considered as a composite if $c_{1}$ and $c_{2}$ are internal and exclusively used by $c_{3}$ and if $r_{3}$ is an internal port of $c_{3}$. According to these changes, a simple drawing shift shown by Figure 12 reveals the well known face of composite components.

$\mathrm{c} 3: \mathrm{C} 3$

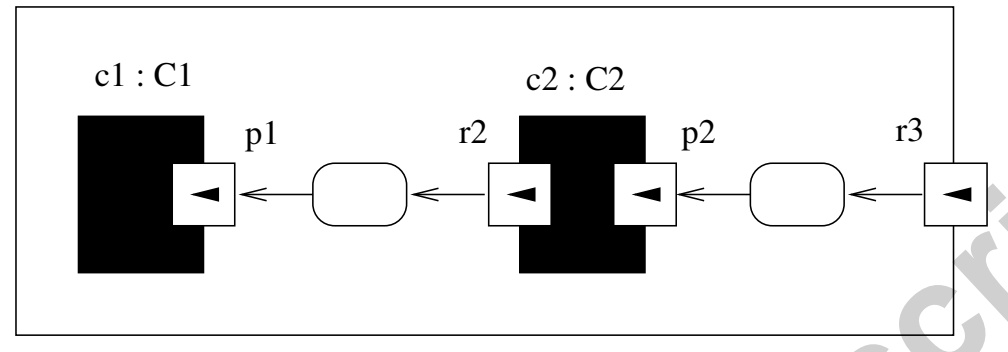

Fig. 11. A composite component

Figure 12 shows the SCL code needed to build the composite component $c_{3}$. The two sub-components $c_{1}$ and $c_{2}$ are instantiated and connected in the init service of $c_{3}$. The init service of a component is always invoked after its instantiation to initialize it. $c_{3}$ has an internal port $r_{3}$ connected to the port $p_{2}$ of $c_{2}$.

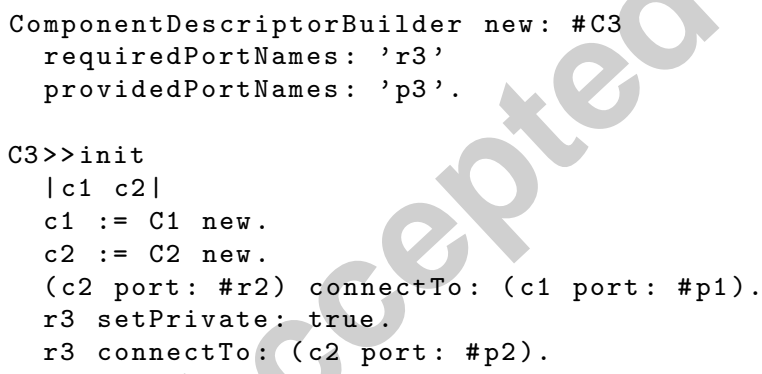

Fig. 12. Definition of a composite component descriptor in SCL

Figure 13 and Figure 14 shows how a composite exports the services provided by its subcomponents. A ForwardConnector is used to forward service invocations received through a provided port to another one. All mismatches problems (e.g name conflicts, adaptation) can be addressed in this regular connector. 
$c: C$

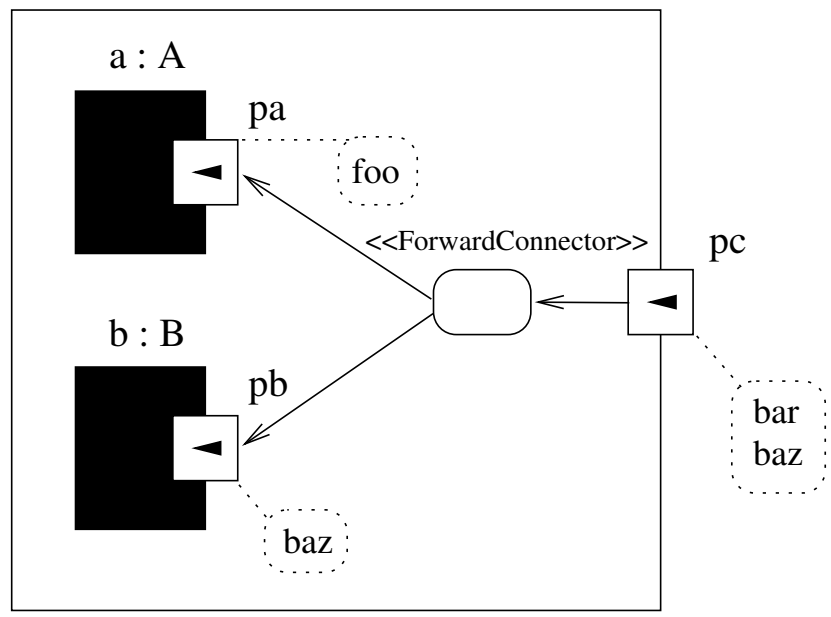

Fig. 13. Service invocation forwarding using a ForwardConNECTOR

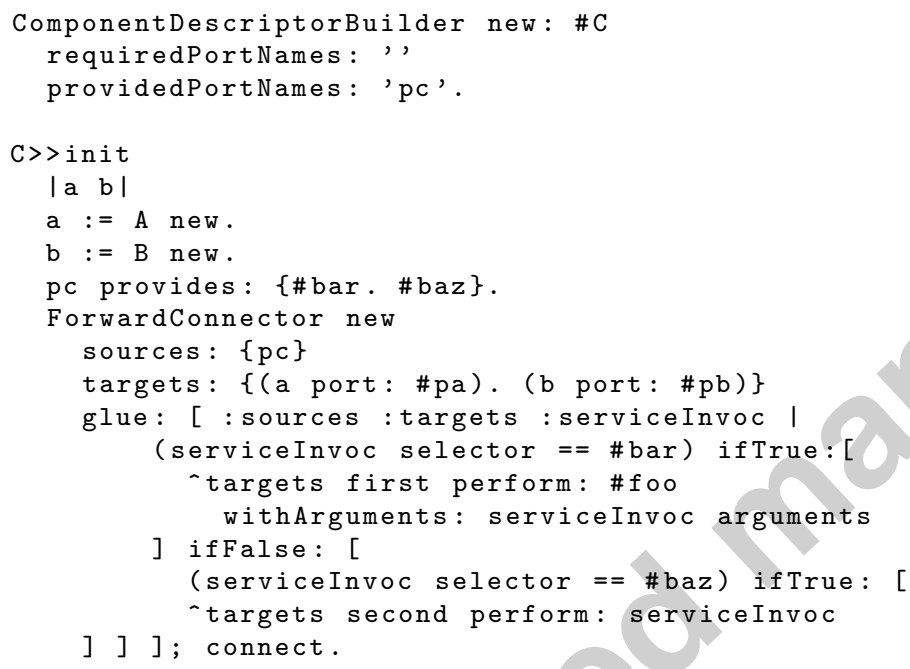

Fig. 14. Using a connector to forward services in a composite component

\section{Separation of concerns in component architectures}

Separation of concerns [44] principle states that a software system should be modularized in such a way that different concerns can be specified as independent as possible in order to maximize understandability and maintainability. Some concerns are difficult to encapsulate in standard software units (components or objects), such as management of transactions, logs, security, etc. To tackle the problem of the scattered code of these concerns, aspect-oriented programming [30] introduces aspects. An aspect is the modularization of a crosscutting concern. Two approaches are distinguished in AOP. Asymmetric approaches, such as AspectJ [29], HyperJ [27] or JAsCo [50], consider aspects as different entities from those ones that compose the base system 
(objects or components). Symmetric approaches, such as Fractal-AOP [17] or FAC [45], try to use the same entities to model the base system and aspects. This second approach is better for reusability because if aspects are modeled as components, they can be used as regular components as well as aspects. A lot of approaches try to merge in a symmetric way aspect-oriented and component-oriented approaches to benefit from the modularity properties of both approaches.

In SCL, we support some aspect-oriented features in a symmetric way. Aspects are regular components and weaving is entirely determined by connections established using special connectors and ports characteristics. The join points - well defined points in the execution of a program where aspects can be woven - are generally $[29,50,17,45]$ method calls, method call receptions, method executions or attribute accesses. All the joint points that are available in aspect-oriented languages built on the top of object-oriented languages, are not suitable for a component-oriented language. For example, in AspectJ, it is possible to specify joint points on private features of a class such as attribute accesses. Since encapsulation is key property of component-oriented languages, we only integrate in SCL the joint points that do not break this property. We choose to support the following join points: before/after/around service invocation receptions by a port or before/after/around the connection/disconnection of a port. Figure 15 shows an example that uses an ADVICECONNECTOR and a regular component 1 to log the service invocations received by our PasswordManager component.

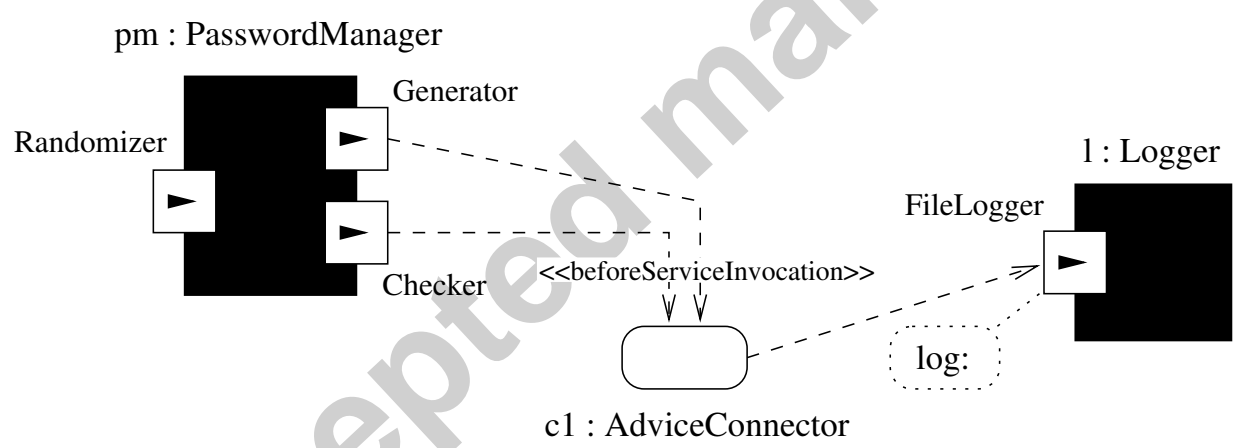

Fig. 15. A LOGGER component used as a crosscuting component using an AdvicECONNECTOR

In an AdviceConnector, each source port is coupled with a keyword (beforeServiceInvocation, beforeConnection, ...). At execution time, when a service invocation arrives on a port, this invocation is transmitted to each of its connected AdviceConnector according to the standard order (around, before, after). A port is able to order its connected connectors since they declare which joint point they are interested in with a keyword. Figure 16 shows the SCL code needed to create the connector $c_{1}$ represented on Figure 15 and also shows the code of a connector $c_{2}$. Thanks to $c_{1}$, the log service of the LOGGER is executed before service invocations through Generator and Checker. Thanks to 
$c_{2}$, the log service of the LOGGER is executed after service invocations through Generator and before those ones through Checker. There is a conflict because multiple glue codes shall be executed before a service invocation on the same port (e.g on line 8 and 16). To prevent these conflicts, we introduce a priority rule stating that the glue code of the last connected connector will be executed first. This priority rule is illustrated on Figure 17 that shows the results given by two provided service invocations of our connected PASSWORDMANAGER. These service invocations supposes that the Randomizer port of the PAssWORDMANAGER has been connected has previously described on Figure 7.

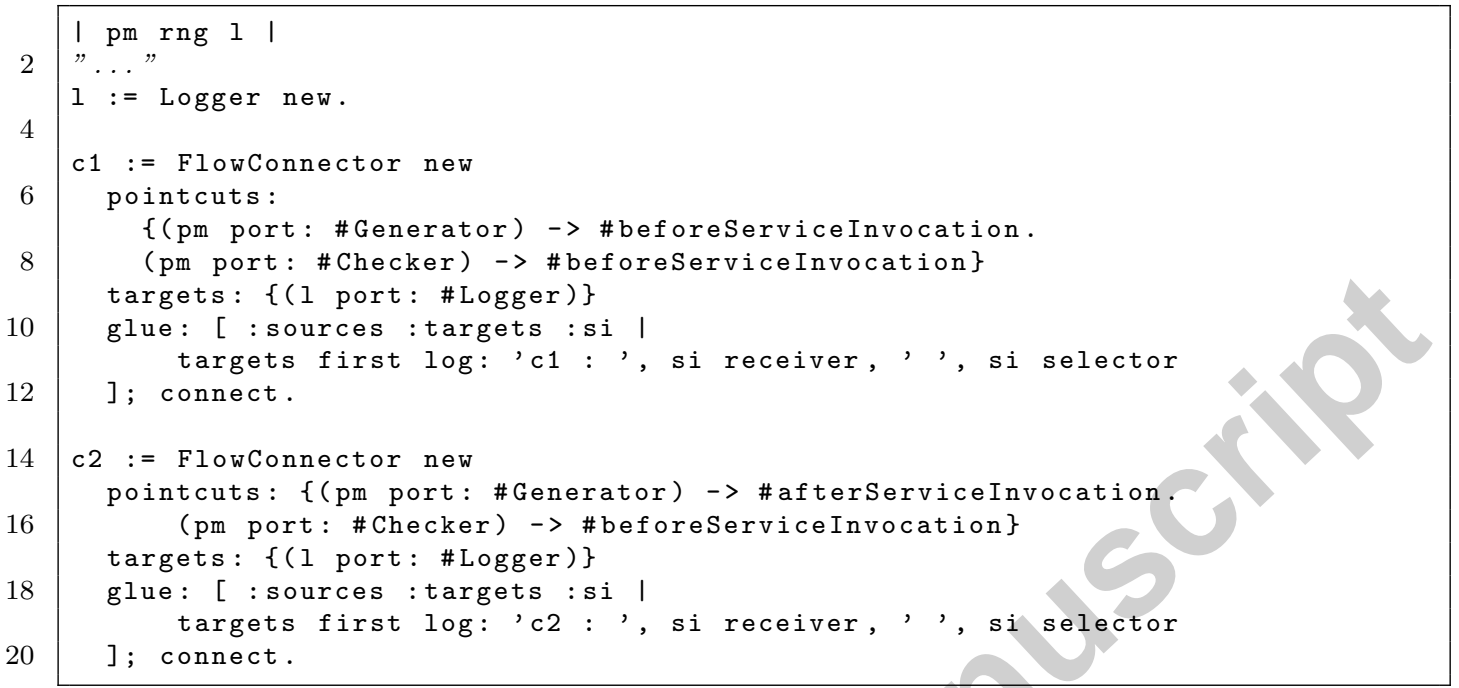

Fig. 16. ScL code of connections that uses the AdviceConnector

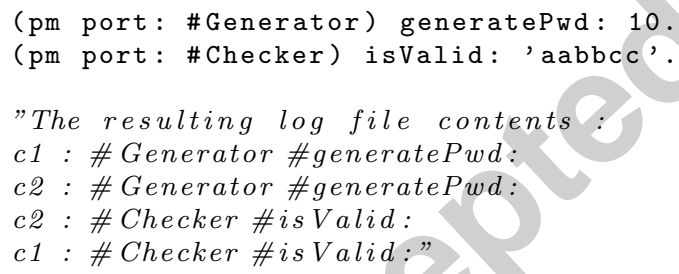

Fig. 17. Two service invocations and their result that illustrate the execution path

\section{Publish/Subscribe communication between components}

Triggering operations as a consequence of state changes in a component is related to Observer design pattern [19] or procedural attachments [38]. In frame languages, it is possible to attach procedures to an attribute access which is then executed each time this attribute is accessed. These kinds of interactions are particularly used between "views" (in the MVC sense [31]) and "models". 
More generally, the publish/subscribe [16] communication protocol is a very useful communication pattern to decouple software entities as said in [20]: "The main invariant in this style is that announcers of events do not know which components will be affected by those events". In component-based languages, this must be done in an unanticipated way and with strict separation between the component code and the connection code to enable components reuse. However, existing proposals fail to solve these two main constraints. Connecting components based on event notifications always require that component programmers add special code in components. We identify the two following problems.

Publishers have to publish events. The component programmer has to add special code such as event signaling in components. For example, in the Java Bean model, the programmer has to manage explicitly the subscribers list (add and remove subscriber methods). In the CCM (Corba Component Model) [42], the component programmer has to manage the event sending by adding a special port to his component that is called an event source, and sends events in the component code through this port. In ArchJava, the component programmer declares broadcast methods (required methods that return void) and invokes them in the component code to signal events. This method is then connected by the architect to multiple provided methods of subscriber components that receive the events. In all cases, the architect can not reuse a component if its programmer has not added special code in the component to signal the event that he needs.

Emitters have to receive events. In the CCM, the component programmer has to provide its components with event sinks that are special ports to receive events. An event sink can be connected by the architect with one or more event sources if they share a compatible event type. This mechanism is more limiting than the ArchJava or the Java Beans one where the subscribers components have only regular methods that are invoked using connections.

In ScL, there are already two ways to enable publish/subscribe connections:

(1) The component programmer integrates the event signaling in the component code. Event signaling in SCL can be done, similarly as in ArchJava, by invoking a required service in the publisher component and regular connections between publishers and subscribers. This solution supposes that the programmer has signaled specific events in the component.

(2) If no event signaling has been integrated by the programmer in the component code, an AdvicConnectors may be used by the architect to detect the events that he needs. For example, if the architect wants to detect when a stack becomes empty (an EmptyStackEvent), he can use an AfterConnector on the port that provides the pop service and test in the glue code if the stack still contains elements to detect such situation. 
If any of the above solution are available, it is not possible for the architect to establish a publish/subscribe connection without modifying the source code of the component. To prevent this issue, we integrate properties in SCL that export the state of a component. This property concept enhances the idea of property of the Javabeans component model [25] with strict separation between the component code and the connection code. For example, a CounTER component has a property named count. This means that it is possible to get and set a value to the count property of the CoUnTER. An example of component with a property is depicted on Figure 18 and the corresponding SCL code is shown on Figure 19.

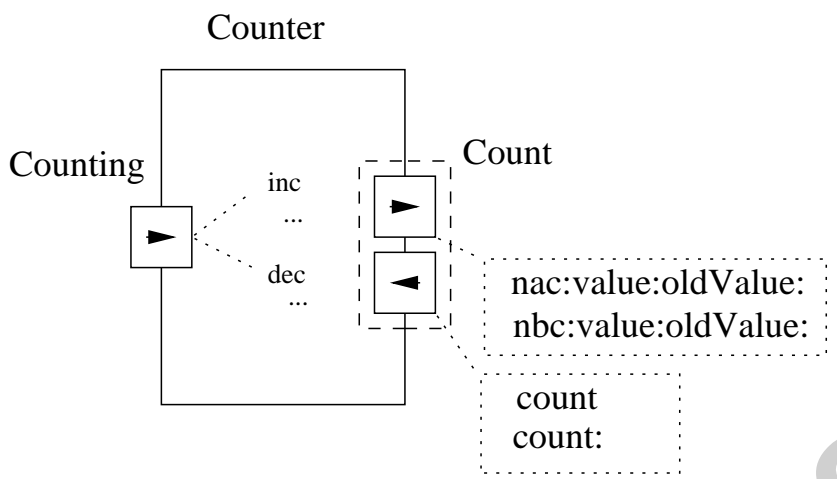

Fig. 18. A Counter component with a value property

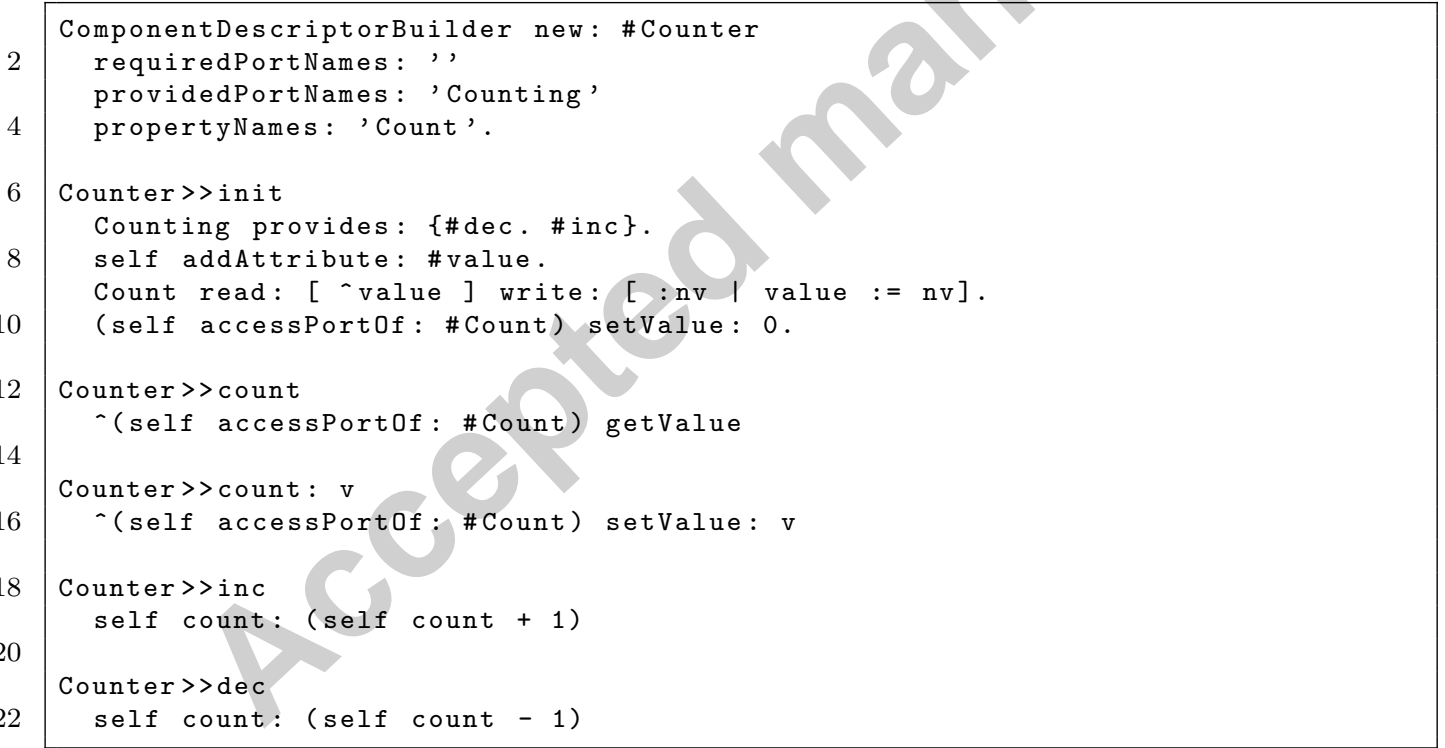

Fig. 19. A Counter component class with a property

When a programmer declares a property, the component is automatically equipped with two ports: an access port and a notifying port. The property access port is a provided port that provides, at least, getter and setter services using the two blocks given during the property declaration (e.g on line 
9 in Figure 19). The notifying port is a required port, which is used to invoke services during property accesses. These services are defined in the SCL component model. For example, the service nac:value:oldValue: (nac is an acronym for Notify After Change) is invoked after a property is modified with the new and the old value of the property as parameters. Another service, the nbc:value:newValue: (nbc is an acronym for Notify Before Change) service, is invoked before the property is modified with the current value and the next value of the property as parameters. In fact, all defined services have two main characteristics: when they are invoked (before or after the property modification) and what a connected component is able to do (nothing, prevent the modification or change the property value). Figure 20 shows the ScL code of two connections $c_{1}$ and $c_{2}$ based on properties notifications. The first connector $c_{1}$ is created using the general syntax form and a BINARYNACCONNECTOR. This connector filters incoming service invocations on the source port and only focuses on the nac:value:oldValue service. After each modification of the value property of the counter, the glue code of the connection is executed and the GUI component is refreshed with the new value (the second parameter of the nac:value:oldValue service). $c_{2}$ achieved the same connection as $c_{1}$ with a less verbose syntax.

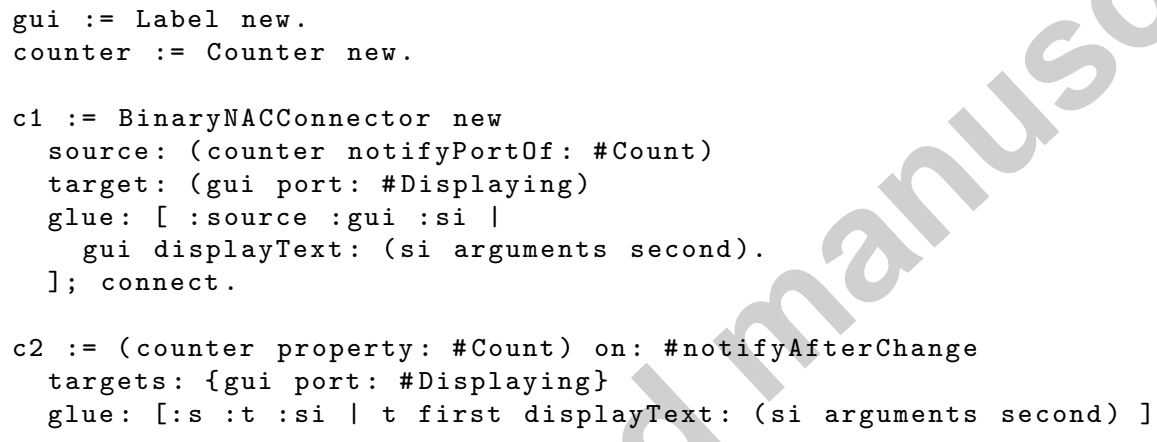

Fig. 20. A connection based on property notifications

SCL provides different kinds of connectors such as BinARYNACCONnECtor, BinarynBCConnector, PropertyBinderConnector ensuring that the value of the target property is always synchronized with the value of the source property. To sum up, component properties are a useful means for component programmers to directly express the external state of components instead of using syntactical conventions and for architects that can use them to connect components.

\section{The Scl Implementation}

The actual prototype of SCL is implemented in Squeak [28]. Squeak is an open and highly portable implementation based on the original Smalltalk- 80 
system [21]. We choose Smalltalk because prototyping is easier and faster than in statically typed languages. It is recognized that dynamic languages offer a lot of advantages [40]. Smalltalk is also a powerful reflective language that enables deep language modifications using message interceptions, addition or modification of meta entities, etc. We also choose Smalltalk because most existing COLs are Java extensions. Using Smalltalk could reveal that existing COLs are more constrained by Java than by the component paradigm.

\subsection{The SCL bootstrap}

The bootstrap of SCL is done with the ComponentDescriptor and Component classes. In the first version, we would like to implement SCL wihtout extending the class concept since we do not want to have some class features (superclass, class organization, ...) in our component descriptors. We created a new bootstrapping kernel by defining Component and ComponentDescriptor as Object subclasses. We succeed to set ComponentDescriptor as the metaclass of Component. It was also necessary to implement our new method (basicNew is defined in the Behavior class). The problem was that all Squeak tools (code browsers, test runner, ...) were not usable with our component descriptors since they were not classes. In the actual prototype, we extend classes to support additional features of component descriptors. We do not use (it is just for squeak compatibility) the features that should not be in component descriptors.

\subsection{The implementation of the SCL model}

Figure 21 shows a simplified scheme reflecting the current implementation of SCL. A component descriptor is represented by a class whose subclass is Component. Component descriptors are instances (indirect instances because of the Smalltalk parallel hierarchy) of a the class named Component class. ComponentDescriptorBuilder is a component, an instance of DEFAULTCOMPONEnTDESCRIPTORBUILDER. All component descriptors are created using the ComponentDescriptorBuilder component which hides that classes are generated. Other features such as ports, interface, property are regular Smalltalk classes. It is not a problem since it is hidden to SCL programmers.

\subsection{Discussion on issues in the current implementation}

The first issue is related to Smalltalk that always enable the programmer to break object encapsulation by using the meta-level. If the SCL programmer 


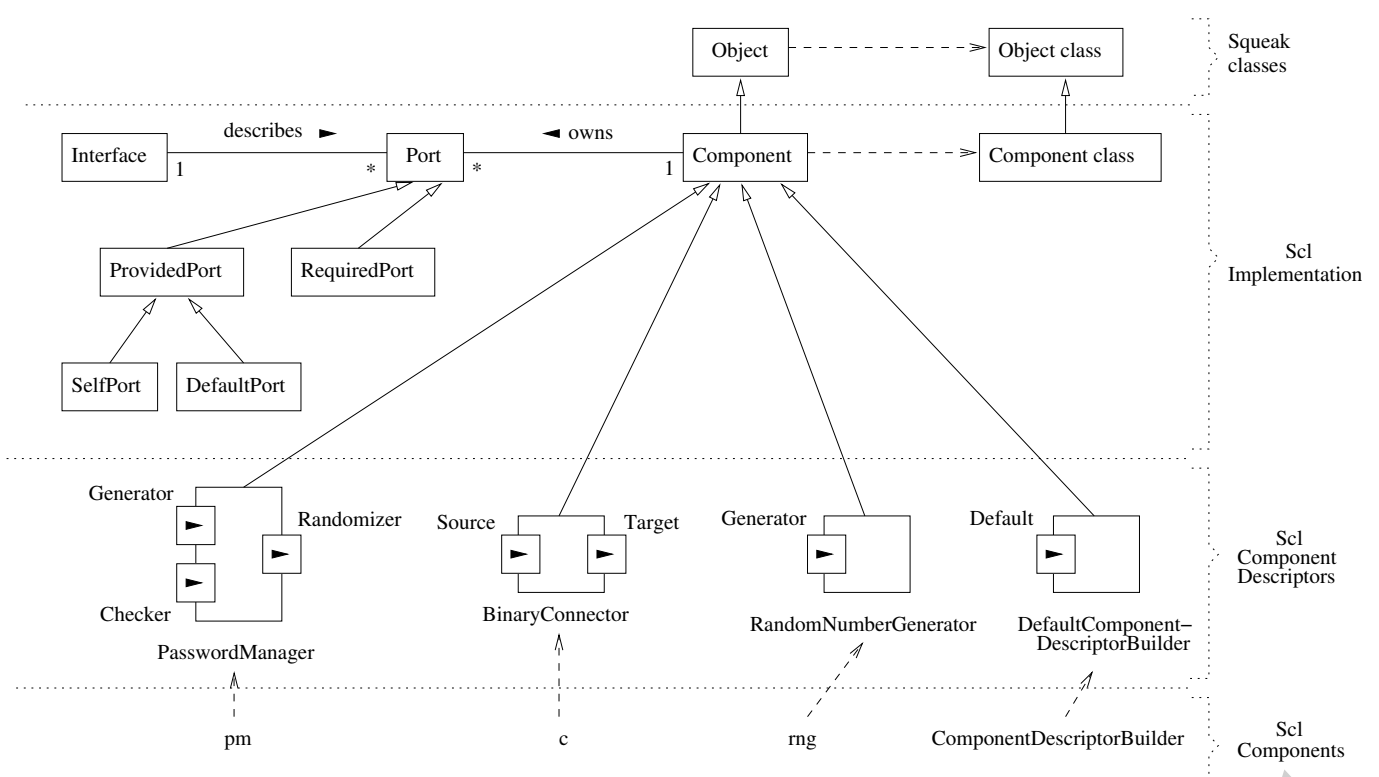

Fig. 21. Overview of the current implementation of SCL

uses Smalltalk methods, he is able to circumvent the SCL mechanisms. However, because we do not implement SCL with an interpreter or a compiler but directly with Smalltalk constructs, it is easier to change and evolve the implementation. This issue could be solved by creating an interpreter for SCL.

The second issue is close to the former one. Since we use the Squeak interpreter, service invocation is achieved using the message sending mechanism. This causes problems when a service invocation is sent through a port. If the service selector of the invocation corresponds to selector of one of the methods defined on the Port class, it is the method that will be executed and not the service of the connected component.

Another potential issue is the efficiency since we have reified all entities of SCL, and also because we do not focus this property. The execution path of a service invocation is the following: (1) the service invocation is received by the port of sending component and transmits it to the connector (it may have more than one connector), (2) the connector execute its glue code and transmits the service invocation to the port of the receiver component, (3) this port transmits the invocation to its component, (4) the component executes the method (6) the result go back through the same way. It is surely non efficient but it is necessary if the glue code do some adaptation. A little optimization is possible if the default glue code (that simply transmit the invocation) is used. In this case, no connector is instantiated and the port of the sending component directly transmit the invocation to the port of the receiver component. 


\section{Synthesis and Related Work}

Short summary of Scl features. A ScL component provides and requires services, receives and sends service invocations through its ports which are described by interfaces. An interface is attached to a port and specifies which services are invokable through this port and the constraints that governs their invocations (service signature compliance, contracts, protocols,... ). Components must be connected through their ports using connectors in order to satisfy their required services. A connector is a component whose role is dedicated to communication between components. There is different kind of connectors such as advice connectors that enable using components as a crosscutting concerns. Components properties represent externalized state of a component and they are the support of connection based on value changes.

SCL is inspired from many existing features of component-oriented languages or models such as ArchJava [2,3], Fractal [9], CCM [42], Java Beans [25], FAC [45], Fractal-AOP [17], ComponentJ [48] and others [33,32,18]. SCL also integrates older ideas such as procedural attachments [38]. Figure 22 shows a graphical synthesis (adapted from [52]) of the main SCL features compared to architectural elements available in some existing models.

composite component (Scl, Archjava, Fractal, ComponentJ)

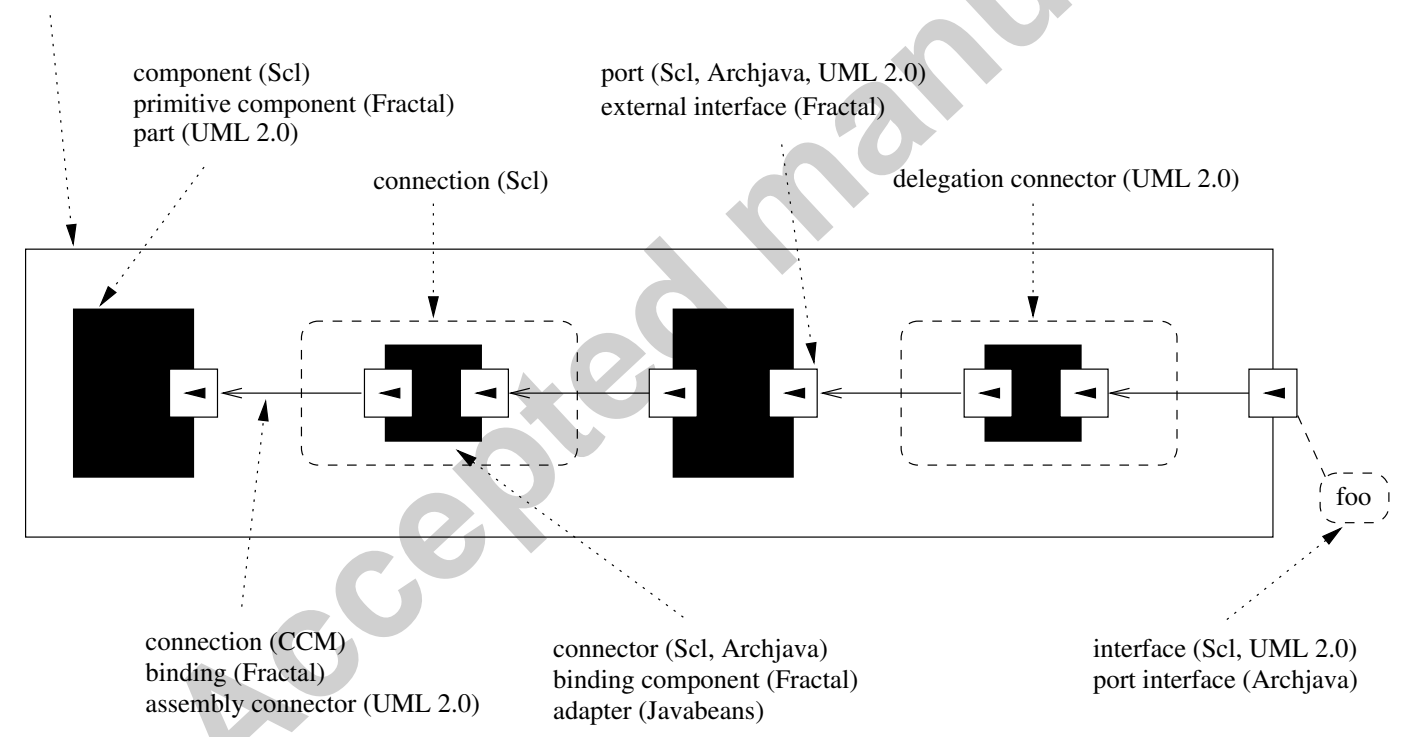

Fig. 22. Graphical comparison between SCL features and existing abstractions in some component models

The work presented in this paper is related to many different research topics:

Component-oriented programming (COP). Many propositions have been done to support COP using object-oriented frameworks or objectoriented language extensions. SCL tries to go a step further with new lan- 
guage with its own abstractions and control structures. There is few work with the same goal such but Lagoona [18] can be considered as one of the first language designed with this objective. Lagoona is based on the idea that modules that contain classes and message definitions are components. Lagoona proposes a new mechanism called generic message forwarding that can be put in relation with service invocation in ScL. However, Lagoona lacks basic component-oriented features (such as port, connector, etc.) and is therefore more restrictive than SCL.

Understanding or teaching COP. Component-oriented programming is not very used compared to object-oriented programming which is actually the dominant paradigm. Although most of COLs are still research prototypes, there is a need to explain, teach and demonstrate what is componentoriented programming using simple and uniform languages as Picolo [33], BoxScript [32] or ScL. Picolo and Boxscript are frameworks respectively written in Python and Java.

Software architecture. Expressing software architecture in terms of connected components is the main objective of architecture description languages (ADLs) [35]. Most of actual features such as component, port, interface, connector have been originally proposed by ADLs. However, most of ADLs are not programming languages. For example, WRIGHT [5] is an ADL based on the formal language called CSP. Although a WRIGHT description is formally defined, it is not executable and must be re-written using a programming language.

Separation of concerns. Separate concerns in different modularity entity is important on the one hand for reuse (a well modularized concern can be reused) and on the other hand for software architecture evolution (there is no scattered code). Separating concerns is difficult either in object-oriented and component-oriented languages. Aspect-oriented languages, such as AspectJ [29] or HyperJ [27] provide a suitable solution to tackle this issue by integrating some new features (aspect, advice, ...) in existing objectoriented language such as Java. Component-oriented languages extensions have also been proposed. Symmetrical approaches, such as FAC [45] or Fractal-AOP [17] seems to be more interesting than others because aspects are regular components. The specificity of SCL is that nothing is written in a component (no special interface has to be implemented). The architect decides to use a component as an aspect component and uses the special connector AdviceConnector. ScL does not support all AOP features because we think that some of them break the component encapsulation. 


\section{Conclusion and future work}

Component-oriented programming will be only possible if new languages are proposed with concepts and mechanisms that enable the unanticipated connection of independently developed software components. In this paper, we have presented SCL a simple, uniform and concrete component-oriented language. The SCL core results from a study of existing languages and of a selection of features that seemed fundamentals. SCL also proposes its own mechanisms such as a general connection mechanism based on connectors. Connectors are useful to solve component connection problems. In SCL, it is possible to create a wide variety of connections: standard required/provided connections, "aspect-like" connections and "publish/subcribe" connections without requiring any code in components thanks to component properties. Properties are declared by the programmer and represent external state of components. A software architect is then able to express connections based on properties notifications.

Ongoing researches on SCL are focused on three areas. First, extending the component model of SCL. For example, what is exactly a port composed of other ports (also known as composite ports or multi-ports)? The answer to this question is important because it could simplify the actual property model and connectors that have sources and targets ports. Second, we plan to provide a faster and more complete version of the prototype of SCL. For example, we are wondering if it is necessary to write an interpreter in order to allow syntax changes between SCL and Smalltalk. In the new version, we want to integrate tools dedicated to component-oriented programming such as a visual component editor. And finally, developing large scale applications using SCL will certainly show us interesting results about the usability of SCL compared to object-oriented languages and the few existing componentoriented languages. This comparison would be interesting but it requires to code the same application in different languages.

\section{References}

[1] Franz Achermann and Oscar Nierstrasz. Applications = Components + Scripts - A Tour of Piccola. In Mehmet Akşit, editor, Software Architectures and Component Technology, pages 261-292. Kluwer, 2001.

[2] Jonathan Aldrich, Craig Chambers, and David Notkin. ArchJava: Connecting Software Architecture to Implementation. In ICSE, pages 187-197. ACM, 2002.

[3] Jonathan Aldrich, Vibha Sazawal, Craig Chambers, and David Notkin. Language support for connector abstractions. In Luca Cardelli, editor, ECOOP, 
volume 2743 of Lecture Notes in Computer Science, pages 74-102. Springer, 2003 .

[4] Robert Allen. A Formal Approach to Software Architecture. PhD thesis, Carnegie Mellon, School of Computer Science, January 1997. Issued as CMU Technical Report CMU-CS-97-144.

[5] Robert Allen and David Garlan. The Wright Architectural Specification Language. Technical report, School of Computer Science, Carnegie Mellon University, Pittsburgh, 1996.

[6] Colin Atkinson, Barbara Paech, Jens Reinhold, and Torsten Sander. Developing and applying component-based model-driven architectures in kobra. edoc, 00:0212, 2001.

[7] Dusan Balek and Frantisek Plasil. Software connectors and their role in component deployment. In Proceedings of DAIS'01, Krakow, Poland, September 2001. Kluwer Academic Publishers.

[8] Manfred Broy, Anton Deimel, Juergen Henn, Kai Koskimies, Frantisek Plasil, Gustav Pomberger, Wolfgang Pree, Michael Stal, and Clemens A. Szyperski. What characterizes a (software) component? Software - Concepts and Tools, 19(1):49-56, 1998.

[9] Eric Bruneton, Thierry Coupaye, Matthieu Leclercq, Vivien Quéma, and JeanBernard Stefani. An Open Component Model and Its Support in Java. In Ivica Crnkovic, Judith A. Stafford, Heinz W. Schmidt, and Kurt C. Wallnau, editors, CBSE, volume 3054 of Lecture Notes in Computer Science, pages 7-22. Springer, 2004.

[10] Martin Büchi and Wolfgang Weck. Compound types for Java. In OOPSLA'98: Proceedings of the 13th ACM SIGPLAN conference on Object-Oriented Programming, Systems, Languages, and Applications, pages 362-373, New York, NY, USA, 1998. ACM Press.

[11] Luca Cardelli. The Handbook of Computer Science and Engineering, chapter 103, Type Systems, pages 2208-2236. CRC Press, Boca Raton, FL, 1997.

[12] John Cheesman and John Daniels. UML components: a simple process for specifying component-based software. Addison-Wesley Longman Publishing Co., Inc., Boston, MA, USA, 2000.

[13] Christophe Dony, Jacques Malenfant, and Pierre Cointe. Prototype-based languages: From a new taxonomy to constructive proposals and their validation. In OOPSLA, pages 201-217, 1992.

[14] Desmond F. D'Souza and Alan Cameron Wills. Objects, components, and frameworks with UML: the catalysis approach. Addison-Wesley Longman Publishing Co., Inc., Boston, MA, USA, 1999.

[15] Michael Eichberg. Mda and programming languages. In Workshop on Generative Techniques in the context of Model Driven Architecture (OOPSLA '02), 2002. 
[16] Patrick Th. Eugster, Pascal A. Felber, Rachid Guerraoui, and Anne-Marie Kermarrec. The many faces of publish/subscribe. ACM Comput. Surv., 35(2):114-131, 2003.

[17] Houssam Fakih, Noury Bouraqadi, and Laurence Duchien. Aspects and software components: A case study of the FRACTAL component model. In Minhuan Huang, Hong Mei, and Jianjun Zhao, editors, International Workshop on Aspect-Oriented Software Development (WAOSD 2004), September 2004.

[18] Peter H. Fröhlich, Andreas Gal, and Michael Franz. Supporting software composition at the programming-language level. Science of Computer Programming, Special Issue on New Software Composition Concept, 56(1-2):4157, April 2005.

[19] Erich Gamma, Richard Helm, Ralph Johnson, and John Vlissides. Design Patterns : Elements of Reusable Object-Oriented Software. Addison Wesley, March 1995.

[20] David Garlan and Mary Shaw. An introduction to software architecture. In V. Ambriola and G. Tortora, editors, Advances in Software Engineering and Knowledge Engineering, pages 1-39, Singapore, 1993. World Scientific Publishing Company.

[21] Adele Goldberg and David Robson. Smalltalk-80: The Language. AddisonWesley Longman Publishing Co., Inc., Boston, MA, USA, 1989.

[22] James Gosling. The Java Language Specification. Addison-Wesley, Boston, 2000 .

[23] Bernhard Gröne, Andreas Knöpfel, and Peter Tabeling. Component vs. component: Why we need more than one definition. In ECBS, pages 550-552. IEEE Computer Society, 2005.

[24] Object Management Group. Uml 2.0 superstructure specification. Technical report, Object Management Group, 2004.

[25] Graham Hamilton. JavaBeans. API Specification, Sun Microsystems, July 1997. Version 1.01.

[26] George T. Heineman and William T. Councill, editors. Component-based software engineering: putting the pieces together. Addison-Wesley Longman Publishing Co., Inc., Boston, MA, USA, 2001.

[27] IBM. Hyper/J. http://www.research.ibm.com/hyperspaces.

[28] Dan Ingalls, Ted Kaehler, John Maloney, Scott Wallace, and Alan Kay. Back to the future: the story of Squeak, a practical Smalltalk written in itself. In OOPSLA '97: Proceedings of the 12th ACM SIGPLAN conference on Objectoriented programming, systems, languages, and applications, pages 318-326, New York, NY, USA, 1997. ACM Press. 
[29] Gregor Kiczales, Erik Hilsdale, Jim Hugunin, Mik Kersten, Jeffrey Palm, and William G. Griswold. An Overview of AspectJ. In Jørgen Lindskov Knudsen, editor, ECOOP, volume 2072 of Lecture Notes in Computer Science, pages 327353. Springer, 2001.

[30] Gregor Kiczales, John Lamping, Anurag Mendhekar, Chris Maeda, Cristina Lopes, Jean-Marc Loingtier, and John Irwin. Aspect-oriented programming. In Mehmet Akşit and Satoshi Matsuoka, editors, 11th Europeen Conf. ObjectOriented Programming, volume 1241 of LNCS, pages 220-242. Springer Verlag, 1997.

[31] Glenn E. Krasner and Stephen T. Pope. A cookbook for using the model-viewcontroller user interface paradigm in smalltalk-80. In Journal of Object-Oriented Programming, volume 1, pages 26-49, Août-Septembre 1988.

[32] Y. Liu and H. C. Cunningham. Boxscript: A component-oriented language for teaching. In 43rd ACM-Southeast Conference, volume 1, pages 349-354, March 2005 .

[33] Raphaël Marvie. Picolo: A simple python framework for introducing component principles. In Euro Python Conference 2005, Göteborg, Sweden, june 2005.

[34] M. D. McIlroy. Mass produced software components. In P. Naur and B. Randell, editors, Proceedings, NATO Conference on Software Engineering, Garmisch, Germany, October 1968.

[35] Nenad Medvidovic and Richard N. Taylor. A classification and comparison framework for software architecture description languages. Software Engineering, 26(1):70-93, 2000.

[36] Nikunj R. Mehta, Nenad Medvidovic, and Sandeep Phadke. Towards a taxonomy of software connectors. In ICSE '00: Proceedings of the 22nd international conference on Software engineering, pages 178-187, New York, NY, USA, 2000. ACM Press.

[37] Microsoft. DCOM technical overview. Microsoft Windows NT Server white paper, Microsoft Corporation, 1996.

[38] M. Minsky. A Framework for Representing Knowledge. In P. Winston, editor, The Psychology of Computer Vision, pages 211-281. mgh, ny, 1975.

[39] Richard Monson-Haefel. Enterprise JavaBeans. O'Reilly \& Associates, Inc., Sebastopol, CA, USA, 1999.

[40] Oscar Nierstrasz, Alexandre Bergel, Marcus Denker, Stéphane Ducasse, Markus Gälli, and Roel Wuyts. On the revival of dynamic languages. In Thomas Gschwind and Uwe Aßmann, editors, Proceedings of Software Composition 2005, volume 3628, pages 1-13. LNCS 3628, 2005. Invited paper.

[41] Oscar Nierstrasz and Laurent Dami. Component-oriented software technology. In Oscar Nierstrasz and Dennis Tsichritzis, editors, Object-Oriented Software Composition, pages 3-28. Prentice-Hall, 1995. 
[42] Object Management Group. Manual of Corba Component Model V3.0, 2002. http://www.omg.org/technology/documents/formal/components.htm.

[43] Object Management Group. Model Driven Architecture, 2003. http://www.omg.org/mda.

[44] D. L. Parnas. On the criteria to be used in decomposing systems into modules. Comm. ACM, 15(12):1053-1058, December 1972.

[45] N. Pessemier, L. Seinturier, L. Duchien, and T. Coupaye. A model for developing component-based and aspect-oriented systems. In Proceedings of the 5th International Symposium on Software Composition (SC'06), volume 4089 of Lecture Notes in Computer Science. Springer, March 2006.

[46] Frantisek Plasil and Stanislav Visnovsky. Behavior protocols for software components. IEEE Trans. Softw. Eng., 28(11):1056-1076, 2002.

[47] Johannes Sametinger. Software engineering with reusable components. SpringerVerlag New York, Inc., New York, NY, USA, 1997.

[48] João Costa Seco and Luís Caires. A basic model of typed components. Lecture Notes in Computer Science, 1850:108-129, 2000.

[49] Mary Shaw. Procedure calls are the assembly language of software interconnection: Connectors deserve first-class status. In ICSE '93: Selected papers from the Workshop on Studies of Software Design, pages 17-32, London, UK, 1996. Springer-Verlag.

[50] Davy Suvée, Wim Vanderperren, and Viviane Jonckers. Jasco: an aspectoriented approach tailored for component based software development. In AOSD '03: Proceedings of the 2nd international conference on Aspect-oriented software development, pages 21-29, New York, NY, USA, 2003. ACM Press.

[51] C. Szyperski. Component Software: Beyond Object-Oriented Programming (2nd Edition). Addison-Wesley, 2002.

[52] Chouki Tibermacine. Contractualisation de l'évolution architecturale de logiciels à base de composants: une approche pour la préservation de la qualité. $\mathrm{PhD}$ thesis, Université de Bretagne Sud, October 2006.

[53] Rob C. van Ommering. Koala, a component model for consumer electronics product software. In Frank van der Linden, editor, ESPRIT ARES Workshop, volume 1429 of Lecture Notes in Computer Science, pages 76-86. Springer, 1998.

[54] Matthias Zenger. Type-safe prototype-based component evolution. In Proceedings of the European Conference on Object-Oriented Programming, Malaga, Spain, June 2002.

[55] Matthias Zenger. Keris: evolving software with extensible modules: Research articles. J. Softw. Maint. Evol., 17(5):333-362, 2005. 